\title{
Two Classes of Photonic Crystals with Simultaneous Band Gaps
}

\author{
Ruey-Lin CHeRN ${ }^{1,2}$, Chien-Chung CHANG $^{2}$, Chien C. ChANG ${ }^{* 2}$ and Robert R. HwANG ${ }^{1,3}$ \\ ${ }^{1}$ Institute of Physics, Academia Sinica, Taipei 115, Taiwan, Republic of China \\ ${ }^{2}$ Institute of Applied Mechanics, National Taiwan University, Taipei 106, Taiwan, Republic of China \\ ${ }^{3}$ Department of System Engineering and Naval Architecture, National Taiwan Ocean University, Keelung 202, Taiwan, Republic of China
}

(Received December 31, 2003; revised February 10, 2004; accepted February 13, 2004; published June 9, 2004)

In this study, we consider band structures of two classes of photonic crystals with two geometric parameters. The first class has a square lattice and is studied for dielectric contrast, centered at $\varepsilon / \varepsilon_{0}=11.4$ (GaAs-air). The second class has a hexagonal lattice and is studied for dielectric contrast, centered at $\varepsilon / \varepsilon_{0}=13$ (silicon-air). These examples have the following feature: the optimal (and largest) full band gap is obtained when both band gaps for $E$ and $H$ polarizations have the same (simultaneous) band edges. In addition, photonic crystals with two geometric parameters typically have much larger optimal band gaps than their counterparts with one geometric parameter. [DOI: 10.1143/JJAP.43.3484]

KEYWORDS: photonic crystals, square lattice, hexagonal lattice, multigrid method, simultaneous band gap

\section{Introduction}

The most attractive/distinguished feature of photonic crystals is their full-band-gap structures. ${ }^{1,2)}$ Large full band gaps allow strong photon localization with the gap, ${ }^{3,4)}$ and a detailed manipulation of photonic defect states. ${ }^{5,6)}$ They have important applications such as in defect cavities, ${ }^{7)}$ optical waveguides, ${ }^{8)}$ defect-mode photonic crystal lasers, ${ }^{9)}$ and feedback mirror in laser diodes. ${ }^{10)}$

Band gap can vary significantly depending on the materials and geometric details of photonic crystals. Considerable effort has been devoted to designing/fabricating photonic structures that possess large band gaps in either $E$ or $H$ polarization or in both. As a result, an empirical rule of thumb $^{11)}$ was formulated from physical principles and numerical experiences: band gaps for $E$ polarization are favored in a lattice of an isolated high- $\varepsilon$ region, and band gaps for $H$ polarization are favored in a connected lattice. The rule is a quite useful guide, but it does not tell when the maximum full band gap will occur. Apparently, there is no general answer to this question. However, in our experience, we need two geometric parameters to balance the band edges of $E$ and $H$ polarizations to obtain the optimal full band gap.

To illustrate the point, let us consider a photonic structure with a hexagonal lattice (denoted as primary), as shown in Fig. 1(a). The maximum full band gap for this hexagonal lattice of circular air columns occurs at $r / a=0.48$ with a gap-midgap ratio of $0.186 .{ }^{11)}$ The corresponding band

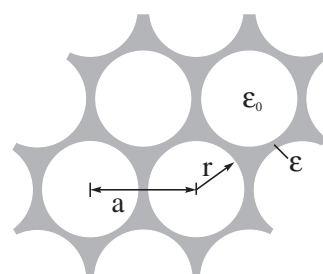

(a)

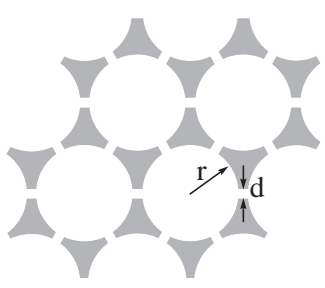

(b)
Fig. 1. (a) Primary hexagonal lattice of circular air columns. (b) Modified hexagonal lattice of circular air columns, each connected to its nearest neighbors by rectangular air rods.

*E-mail address: changcc@gauss.iam.ntu.edu.tw

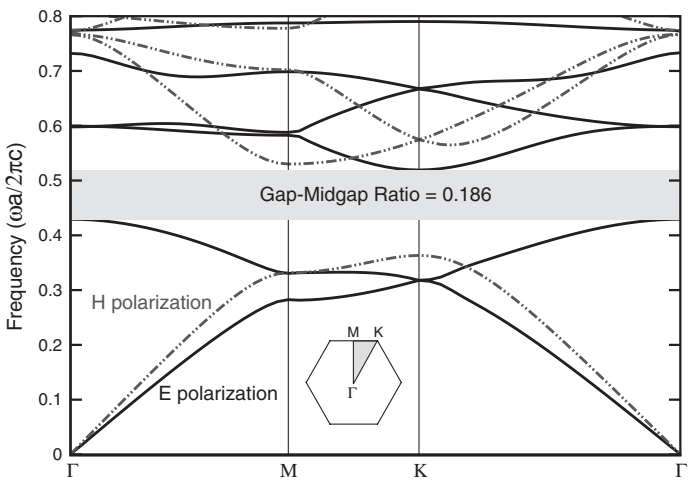

Fig. 2. Band structures computed with $256 \times 256$ grid for the primary hexagonal lattice in Fig. 1(a). The maximum band gap is attained at $r / a=0.48$ for $\varepsilon / \varepsilon_{0}=13$.

structure is plotted in Fig. 2, which shows, however, that the band gap for $H$ polarization is much wider than that for $E$ polarization. Figure 1(b) shows that the above structure can be easily modified by connecting each air column to its nearest neighbors with slender rectangular air rods. Note that the modified structure has two geometric parameters, $r / a$ and $d / a$. Numerical results show that the largest full band gap occurs at $r / a=0.48$ and $d / a=0.045$ with a larger gapmidgap ratio of 0.2097 . The corresponding band structure is shown in Fig. 3. It is of particular interest to note that at this

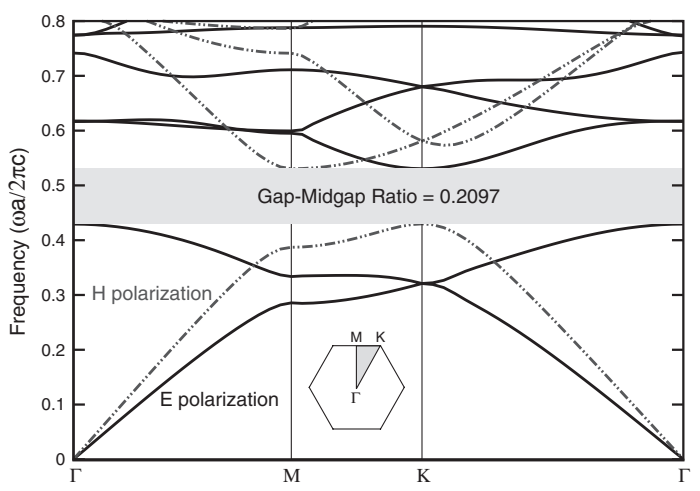

Fig. 3. Band structures computed with $256 \times 256$ grid for the modified hexagonal lattice in Fig. 1(b). The optimal band gap is attained at $r / a=$ 0.48 and $d / a=0.045$ for $\varepsilon / \varepsilon_{0}=13$. 
Table I. Comparison of the band edges for $E$ and $H$ polarizations and their relative differences for the primary and modified photonic structures in Fig. 1 with $\varepsilon / \varepsilon_{0}=13$.

\begin{tabular}{ccc}
\hline $\begin{array}{c}\text { shape } \\
\text { mode }\end{array}$ & $\begin{array}{c}\text { primary } \\
\text { TM/TE }\end{array}$ & $\begin{array}{c}\text { modified } \\
\text { TM/TE }\end{array}$ \\
\hline$a \omega_{\text {up }} / 2 \pi c$ & $0.5192 / 0.5301$ & $0.5305 / 0.5305$ \\
$a \omega_{\text {low }} / 2 \pi c$ & $0.4293 / 0.3633$ & $0.4294 / 0.4298$ \\
$\alpha_{\text {up }}$ & $2.1 \%$ & $0 \%$ \\
$\beta_{\text {low }}$ & $17 \%$ & $0.093 \%$ \\
\hline
\end{tabular}

optimal band gap, both $E$ and $H$ polarizations now have the same band edges, namely, they have a simultaneous band gap. In addition, the modification in geometry does not change the branches of frequency, between which the full band gap occurs.

For a detailed comparison, Table I lists the numerics of the upper and lower band edges of the primary and modified hexagonal lattices. Let $\omega_{\text {up }}$ denote the upper edge, $\omega_{\text {low }}$ the lower edge, and $\alpha_{\text {up }}$ the relative difference between the upper edges of the band gaps for $E(T M)$ and $H(T E)$ polarizations, and $\beta_{\text {low }}$ the relative difference between the lower edges. The relative difference in the upper edge $\alpha_{\text {up }}$ drops from $2.1 \%$ of the primary structure to $0 \%$ of the modified, while the relative difference in the lower edge $\beta_{\text {low }}$ drops significantly from $17 \%$ to $0.093 \%$. Regarding band gap size, we find a $12.7 \%$ increase in the gap-midgap ratio of the full band gap from 0.186 of the primary structure to 0.2097 of the modified structure. In summary, the modified structure has two salient features: (i) the optimal full band gap occurs as a simultaneous band gap in both $E$ and $H$ polarizations, and (ii) the full band gap is substantially larger than that of the primary lattice. Point (ii) can be reasonably expected because the modified structure has two parameters rather than one, but the coincidence in point (i) is not apparent and is worthy of further investigation. An ambitious goal would be to investigate the validity of the proposition: If two (or more) geometric parameters are properly chosen, the optimal full band gap is obtained when both band gaps for $E$ and $H$ polarizations have the same (simultaneous) band edges. The present study does not completely answer the above question, but illustrates by examples such possibilities in the sense that the proposition is approximately valid, or that photonic structures with one geometric parameter may be easily modified to have two geometric parameters so that the proposition holds within acceptable tolerance.

For this purpose, we propose to investigate a class of photonic structures with a square lattice and another class with a hexagonal lattice, both with two geometric parameters. Figure 4 shows three types of square lattice: one with circular dielectric columns (SCR), and two with square columns (SS1, SS2). Notice the distinction that the rods connect the sides of the columns in type SS1, and vertices in type SS2. Figure 5 shows five types of hexagonal lattice: one with circular dielectric columns (HCR), two with hexagonal columns (HEX1, HEX2) and another two with triangular columns (TRI1, TRI2). Both HEX1 and HEX2 have hexagonal columns. The only distinction is whether the rods connect the sides or the vertices of the columns. Likewise, TRI1 and TRI2 have triangular columns with rods

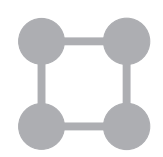

(a)SCR

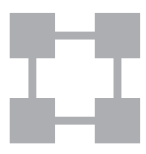

(b)SS1

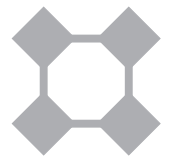

(c)SS2
Fig. 4. Three types of square lattice with dielectric columns and connecting rods: (a) SCR (b) SS1 (c) SS2.

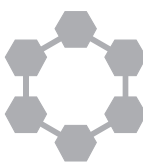

(b)HEX1

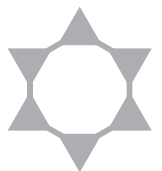

(d)TRI1

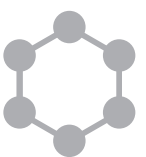

(a) $\mathrm{HCR}$

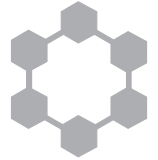

(c)HEX2

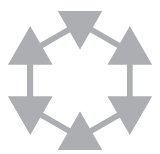

(e)TRI2
Fig. 5. Five types of hexagonal lattice with dielectric columns and connecting rods: (a) HCR (b) HEX1 (c) HEX2 (d) TRI1 (d) TRI2.

connecting the vertices and sides of the columns, respectively. They are constructed to have same group of symmetry, and all of them will be shown to have large full band gaps when optimized by balancing the band gaps of $E$ and $H$ polarizations.

Photonic structures with two or more adjustable parameters require much more computational resources to search for the largest (optimal) full band gaps. In the present study, all the band structures with the first few branches of eigenfrequency (including those in Fig. 1 and Table I) can be computed efficiently by an inverse method with multigrid acceleration, which has recently been developed by the present authors. ${ }^{12}$ ) The method is capable of resolving multieigenvalue band structures, and is applicable to photonic crystals with interfaces of strong contrast.

\section{Basic Equations and Formulation}

Electromagnetic waves propagating in photonic crystals are well-described by Maxwell's equations. For linear isotropic and frequency-independent dielectric materials with permeability close to one, the time-harmonic modes of electromagnetic waves for $E$ polarization $(T M)$ can be written as

$$
-\frac{1}{\varepsilon}\left(\frac{\partial^{2}}{\partial x^{2}}+\frac{\partial^{2}}{\partial y^{2}}\right) E=\left(\frac{\omega}{c}\right)^{2} E
$$

and for $H$ polarization $(T E)$ as

$$
-\left[\frac{\partial}{\partial x}\left(\frac{1}{\varepsilon} \frac{\partial}{\partial x}\right)+\frac{\partial}{\partial y}\left(\frac{1}{\varepsilon} \frac{\partial}{\partial y}\right)\right] H=\left(\frac{\omega}{c}\right)^{2} H,
$$


where $E$ and $H$ are the scalar electric and magnetic field intensities, respectively, $\varepsilon=\varepsilon(\boldsymbol{r})$ is the dielectric function, $\omega$ is the angular frequency of the time-harmonic modes, and $c$ is the speed of light in vacuum. To discretize eqs. (1) and (2), a second-order central finite difference scheme is used. As to the treatment of the interface of the dielectric function, there are several possibilities. One simple idea is to interpolate the dielectric function at the interface. ${ }^{13,14)}$ At the grid point near the interface, the dielectric function is expressed as

$$
\varepsilon=f_{a} \varepsilon_{a}+f_{b} \varepsilon_{b}
$$

for $E$ polarization and

$$
\frac{1}{\varepsilon}=f_{a} \frac{1}{\varepsilon_{a}}+f_{b} \frac{1}{\varepsilon_{b}}
$$

for $H$ polarization, where $\varepsilon_{a}$ and $\varepsilon_{b}$ are the dielectric constants of materials $a$ and $b$, and $f_{a}$ and $f_{b}$ are the fractions of the grid cell that contain $\varepsilon_{a}$ and $\varepsilon_{b}$, respectively.

The domain of computation for the square lattice is shown in Fig. 6. For the hexagonal lattice, the domain of computation is chosen as a rectangle with the same area of the primitive cell, as shown in Fig. 7. Bloch's theorem is applied at the domain boundary.

$$
\begin{aligned}
& E_{\boldsymbol{k}}\left(\boldsymbol{r}+\boldsymbol{a}_{i}\right)=e^{i \boldsymbol{k} \cdot \boldsymbol{a}_{i}} E_{\boldsymbol{k}}(\boldsymbol{r}) \\
& H_{\boldsymbol{k}}\left(\boldsymbol{r}+\boldsymbol{a}_{i}\right)=e^{i \boldsymbol{k} \cdot \boldsymbol{a}_{i}} H_{\boldsymbol{k}}(\boldsymbol{r})
\end{aligned}
$$

where $E_{k}$ and $H_{k}$ are Bloch's functions for electric and magnetic fields, respectively, associated with the wave vector $\boldsymbol{k}$ in the first Brillouin zone, and $\boldsymbol{a}_{i}(i=1,2)$ are lattice translation vectors. For the square lattice, $\boldsymbol{a}_{1}=a(1,0)$ and $\boldsymbol{a}_{2}=a(0,1)$. For the hexagonal lattice, $\boldsymbol{a}_{1}=a(1,0)$ and

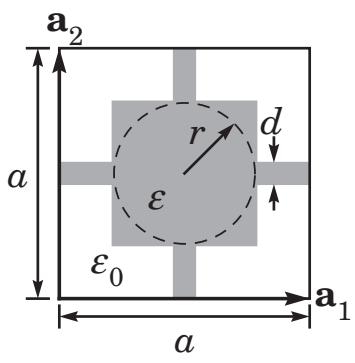

Fig. 6. The domain of computation for the square lattice has width $a$ and height $a$. The radius of the incircle of the dielectric column is $r$ and the width of the dielectric connecting rod is $d$.

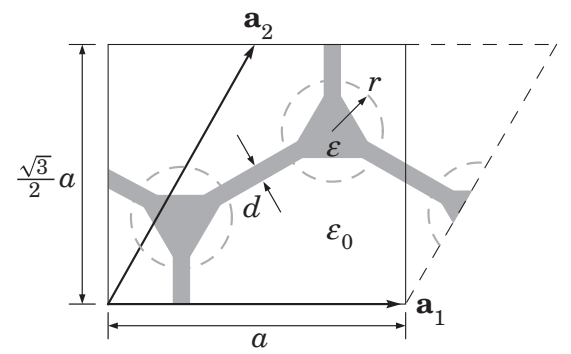

Fig. 7. The domain of computation for the hexagonal lattice has width $a$ and height $\sqrt{3} a / 2$. The radius of the circumcircle of the dielectric column is $r$ and the width of the dielectric connecting rod is $d$. $\boldsymbol{a}_{2}=a(1 / 2, \sqrt{3} / 2)$. In the latter case, since $\boldsymbol{a}_{2}$ forms a $60^{\circ}$ angle with the $x$-axis, the application of Bloch's condition in the $y$-direction should be additionally accompanied by onehalf cell in the $x$-direction.

The eigenvalue problem to be solved consists of eqs. (1) and (5) or eqs. (2) and (6). Both of them can be put in the dispersion-relation form

$$
D\left(a \omega / 2 \pi c, k a ; r / a, d / a, \varepsilon / \varepsilon_{0}\right)=0 .
$$

For a specified geometry and dielectric constants, we solve for the first ten branches of eigenfrequency $a \omega / 2 \pi c$ in terms of the wave number $\boldsymbol{k} a$ belonging to the first Brillouin zone. The method of solution used here is the inverse method with multigrid acceleration as mentioned in $\S 1$.

\section{Results and Discussion}

As an example of a test, we compute the first eight frequency bands for $E$ polarization at the symmetric point $\mathrm{K}$ in the first Brillouin zone for the five types of hexagonal lattice in Fig. 5 with $\varepsilon / \varepsilon_{0}=13$. Table II shows the computation times in CPU seconds for these configurations. Computations are performed on a Pentium $4-2.8 \mathrm{GHz}$ PC. The number of grid points $N=N_{\text {grid }}$ ranges from $64^{2}$ to $1024^{2}$. The computation times for the present method are better than that on the order $N$. If the operation counts are fitted with $N^{\alpha}$, we find the values of $\alpha$ for types HCR, HEX1, HEX2, TRI1 and TRI2 to be 0.7242, 0.7305, 0.7322, 0.7693 and 0.7529 , respectively. They are surprisingly better orders than linear $N$. This is mainly because the present method interlaces an inverse method and multigrid acceleration, making the present method superlinear in operation counts.

To illustrate the convergence of the computed results against grid size, computations are performed on five different grids: $N=64^{2}, 128^{2}, 256^{2}, 512^{2}$, and $1024^{2}$. Table III lists the numerics of the location of the maximum full band gap for the type HCR in Fig. 5(a) with $\varepsilon / \varepsilon_{0}=13$. Basically, all the computed results of the band edges $\left(\omega_{\text {up }}\right.$, $\left.\omega_{\text {low }}\right)$, midgap frequency $\left(\omega_{\text {mid }}\right)$, band width $(\Delta \omega)$ and gap-

Table II. Computation times in CPU seconds for eight bands of a single Bloch wave number and order of operation count $N^{\alpha}$ for different configurations in Fig. 5 with $\varepsilon / \varepsilon_{0}=13$.

\begin{tabular}{lcccccc}
\hline \multicolumn{1}{c}{$N$} & $64^{2}$ & $128^{2}$ & $256^{2}$ & $512^{2}$ & $1024^{2}$ & $\alpha$ \\
\hline HCR & 0.531 & 2.13 & 6.39 & 20.4 & 62.1 & 0.7242 \\
HEX1 & 0.516 & 2.05 & 6.52 & 20.3 & 61.9 & 0.7305 \\
HEX2 & 0.516 & 2.13 & 6.44 & 20.3 & 64.8 & 0.7322 \\
TRI1 & 0.437 & 1.48 & 5.39 & 18.6 & 60.4 & 0.7693 \\
TRI2 & 0.562 & 1.66 & 5.59 & 20.1 & 68.2 & 0.7529 \\
\hline
\end{tabular}

Table III. Convergence of the band edges of the full band gap for type HCR in Fig. 5(a) with $\varepsilon / \varepsilon_{0}=13$ against grid size. $\omega_{\text {mid }}=\left(\omega_{\text {up }}+\omega_{\text {low }}\right) / 2$ and $\Delta \omega=\omega_{\text {up }}-\omega_{\text {low }}$.

\begin{tabular}{cccccc}
\hline$N$ & $64^{2}$ & $128^{2}$ & $256^{2}$ & $512^{2}$ & $1024^{2}$ \\
\hline$a \omega_{\text {up }} / 2 \pi c$ & 0.4917 & 0.4940 & 0.4940 & 0.4941 & 0.4940 \\
$a \omega_{\text {low }} / 2 \pi c$ & 0.3946 & 0.3876 & 0.3873 & 0.3873 & 0.3873 \\
$a \omega_{\text {mid }} / 2 \pi c$ & 0.4431 & 0.4408 & 0.4407 & 0.4407 & 0.4407 \\
$a \Delta \omega / 2 \pi c$ & 0.0971 & 0.1064 & 0.1067 & 0.1068 & 0.1067 \\
$\Delta \omega / \omega_{\text {mid }}$ & 0.2191 & 0.2413 & 0.2421 & 0.2423 & 0.2421 \\
\hline
\end{tabular}


Table IV. Band edges and their relative differences for $E(T M)$ and $H(T E)$ polarizations for the three types of photonic structure in Fig. 4 with $\varepsilon / \varepsilon_{0}=11.4$.

\begin{tabular}{lccc}
\hline $\begin{array}{l}\text { shape } \\
\text { mode }\end{array}$ & $\begin{array}{c}\text { SCR } \\
\text { TM/TE }\end{array}$ & $\begin{array}{c}\text { SS1 } \\
\text { TM/TE }\end{array}$ & $\begin{array}{c}\text { SS2 } \\
\text { TM/TE }\end{array}$ \\
\hline$a \omega_{\text {up }} / 2 \pi c$ & $0.48 / 0.4793$ & $0.4607 / 0.459$ & $0.4894 / 0.4897$ \\
$a \omega_{\text {low }} / 2 \pi c$ & $0.4031 / 0.4035$ & $0.3929 / 0.3997$ & $0.4118 / 0.4136$ \\
$\alpha_{\text {up }}$ & $0.15 \%$ & $0.37 \%$ & $0.061 \%$ \\
$\beta_{\text {low }}$ & $0.099 \%$ & $1.7 \%$ & $0.44 \%$ \\
\hline
\end{tabular}

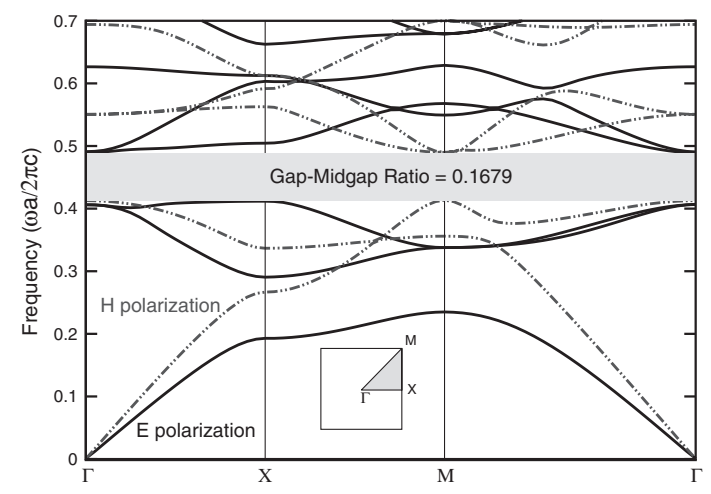

Fig. 8. Band structures computed with $256 \times 256$ grid for type SS2 in Fig. 4(c). The optimal band gap is attained at $r / a=0.275$ and $d / a=$ 0.03 with $\varepsilon / \varepsilon_{0}=11.4$.

midgap ratio $\left(\Delta \omega / \omega_{\text {mid }}\right)$ for the grid $N_{\text {grid }} \geq 128 \times 128$ coincide with each other to three significant digits. The table indicates that the grid size $128 \times 128$ is appropriate for the present study. More details about the present method, including the algorithms and comparison with plane wave expansions (PWE) can be found in ref. 14.

First of all, we consider the three types of square lattice shown in Fig. 4 with a dielectric contrast $\varepsilon / \varepsilon_{0}$ of 11.4 (GaAs-air). All the full band gaps are optimized with respect to $r / a$ and $d / a$. Table IV shows the numerics of the upper and lower edges of the band gaps for $E$ and $H$ polarizations, and their relative differences in edge location. The coincidence of the band edges is quite well, and all the differences are within $2 \%$. In particular, Fig. 8 shows the corresponding band structure for type SS2. It is seen that the band edges for both polarizations occur at different symmetric points. $H$ polarization has the upper edge at the symmetric point $M$, and the lower at the symmetric point $\Gamma$, while $E$ polarization has the upper edge at the symmetric point $\Gamma$, and the lower at the symmetric point $X$. Namely, the band gaps for $E$ and $H$ polarizations balance well with each other, even though their individual band edges occur at different symmetric points. Actually, all the three types of square lattice in Fig. 4 have the same edge Bloch wave numbers: $M-\Gamma$ (upper-lower) for $H$ polarization, and $\Gamma-X$ (upper-lower) for $E$ polarization. As the location of the band gap in the domain of frequency is concerned, the full band gap for all the three types lies between the 2nd and 3rd branches for $H$ polarization and between the 3rd and 4th branches for $E$ polarization.

Next, we consider the five types of hexagonal lattice shown in Fig. 5 with a dielectric contrast $\varepsilon / \varepsilon_{0}$ of 13 (siliconair). Again, all the full band gaps are optimized with respect to $r / a$ and $d / a$. Table $\mathrm{V}$ shows the numerics of the upper
Table V. Band edges and their relative differences for $E(T M)$ and $H(T E)$ polarizations for the five types of photonic structure in Fig. 5 with $\varepsilon / \varepsilon_{0}=13$.

\begin{tabular}{ccc}
\hline shape & HCR & HEX1 \\
mode & TM/TE & TM/TE \\
\hline$a \omega_{\text {up }} / 2 \pi c$ & $0.4949 / 0.494$ & $0.4942 / 0.4949$ \\
$a \omega_{\text {low }} / 2 \pi c$ & $0.3873 / 0.3843$ & $0.3874 / 0.3853$ \\
$\alpha_{\text {up }}$ & $0.18 \%$ & $0.14 \%$ \\
$\beta_{\text {low }}$ & $0.78 \%$ & $0.54 \%$ \\
\hline HEX2 & TRI1 & TRI2 \\
TM/TE & TM/TE & TM/TE \\
\hline $0.4896 / 0.4904$ & $0.5119 / 0.51$ & $0.5087 / 0.5088$ \\
$0.3847 / 0.3806$ & $0.3993 / 0.3987$ & $0.4093 / 0.3945$ \\
$0.16 \%$ & $0.37 \%$ & $0.02 \%$ \\
$1.1 \%$ & $0.15 \%$ & $3.7 \%$ \\
\hline
\end{tabular}

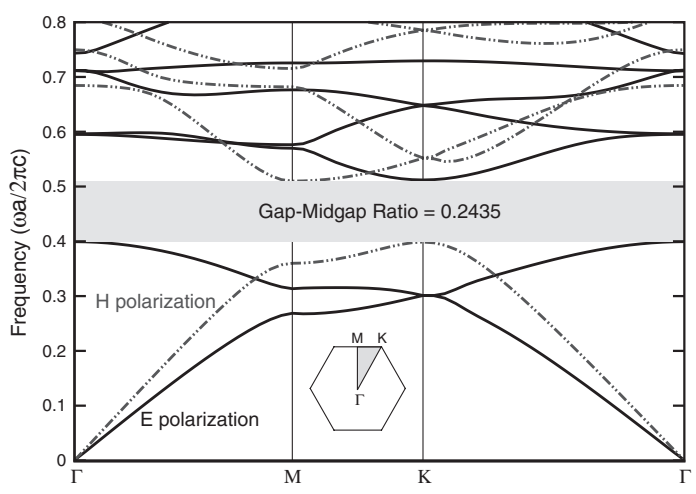

Fig. 9. Band structures computed with $256 \times 256$ grid for type TRI1 in Fig. 5(d). The optimal band gap is attained at $r / a=0.245$ and $d / a=$ 0.015 with $\varepsilon / \varepsilon_{0}=13$.

and lower edges of the band gaps for $E$ and $H$ polarizations, and their relative differences in edge location. The coincidence of the band edges are quite well, and all the errors are within $1.1 \%$, except for $3.7 \%$ for the lower edge for type TRI2. In particular, Fig. 9 shows the corresponding band structure for type TRI1. Again, the band edges for both polarizations occur at different symmetric points. $H$ polarization has the upper edge at the symmetric point $M$, and the lower at the symmetric point $K$, while $E$ polarization has the upper edge at the symmetric point $K$, and the lower at the symmetric point $\Gamma$. The band edges for $E$ and $H$ polarizations do not occur at the same place, but they do balance with each other to yield the optimal (and the largest) band gaps (with respect to the geometric parameters $d / a$ and $r / a$ ). The same as that in the case of the square lattice, all the five types of hexagonal lattice in Fig. 5 have the same edge Bloch wave numbers: $M-K$ (upper-lower) for $H$ polarization, and $K-\Gamma$ (upper-lower) for $E$ polarization. As the location of the band gap in the domain of frequency is concerned, the full band gap for all the five types lies between the 1 st and 2 nd branches for $H$ polarization and between the 2 nd and $3 r$ branches for $E$ polarization.

There is one more aspect worthy of investigation, i.e., the effect of the dielectric contrast $\left(\varepsilon / \varepsilon_{0}\right)$, which actually provides one additional parameter. For the square lattice of the type $\mathrm{SS} 2$, we vary the dielectric contrast $\left(\varepsilon / \varepsilon_{0}\right)$ from 10.5 to 12.5 , centered at 11.4 , and the results for the optimal full 
Table VI. Variation of band edges and their relative differences with dielectric contrast for type SS2 in Fig. 4(c).

\begin{tabular}{|c|c|c|c|c|c|}
\hline $\begin{array}{l}\varepsilon / \varepsilon_{0} \\
\text { mode }\end{array}$ & $\begin{array}{c}10.5 \\
\mathrm{TM} / \mathrm{TE}\end{array}$ & $\begin{array}{c}11 \\
\mathrm{TM} / \mathrm{TE}\end{array}$ & $\begin{array}{c}11.4 \\
\mathrm{TM} / \mathrm{TE}\end{array}$ & $\begin{array}{c}12 \\
\mathrm{TM} / \mathrm{TE}\end{array}$ & $\begin{array}{c}12.5 \\
\mathrm{TM} / \mathrm{TE}\end{array}$ \\
\hline$a \omega_{\text {up }} / 2 \pi c$ & $0.4876 / 0.4831$ & $0.4889 / 0.488$ & $0.48 / 0.4793$ & $0.4781 / 0.4787$ & $0.468 / 0.4687$ \\
\hline$\alpha_{\text {up }}$ & $0.53 \%$ & $0.34 \%$ & $0.099 \%$ & $0.3 \%$ & $1.2 \%$ \\
\hline$\beta_{\text {low }}$ & $0.93 \%$ & $0.18 \%$ & $0.15 \%$ & $0.13 \%$ & $0.15 \%$ \\
\hline
\end{tabular}

Table VII. Variation of band edges and their relative differences with dielectric contrast for type TRI1 in Fig. 5(d).

\begin{tabular}{|c|c|c|c|c|c|}
\hline $\begin{array}{l}\varepsilon / \varepsilon_{0} \\
\text { mode }\end{array}$ & $\begin{array}{c}12 \\
\mathrm{TM} / \mathrm{TE}\end{array}$ & $\begin{array}{c}12.5 \\
\mathrm{TM} / \mathrm{TE}\end{array}$ & $\begin{array}{c}13 \\
\mathrm{TM} / \mathrm{TE}\end{array}$ & $\begin{array}{c}13.5 \\
\mathrm{TM} / \mathrm{TE}\end{array}$ & $\begin{array}{c}14 \\
\mathrm{TM} / \mathrm{TE}\end{array}$ \\
\hline$a \omega_{\mathrm{up}} / 2 \pi c$ & $0.4923 / 0.4920$ & $0.5027 / 0.5018$ & $0.4949 / 0.4940$ & $0.4981 / 0.4983$ & $0.5016 / 0.5025$ \\
\hline$\alpha_{\text {up }}$ & $0.061 \%$ & $0.18 \%$ & $0.18 \%$ & $0.04 \%$ & $0.18 \%$ \\
\hline$\beta_{\text {low }}$ & $4.6 \%$ & $4.5 \%$ & $0.78 \%$ & $0.39 \%$ & $0.29 \%$ \\
\hline
\end{tabular}

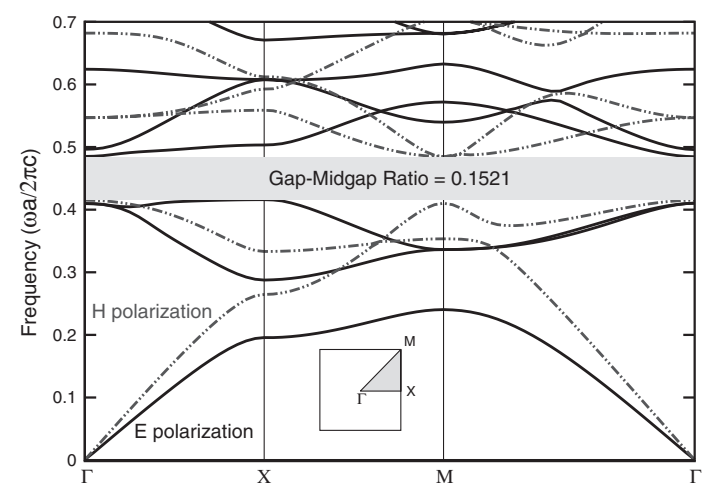

Fig. 10. Band structures computed with $256 \times 256$ grid for type SS2 in Fig. 4(c). The optimal band gap is attained at $r / a=0.29$ and $d / a=0.03$ with $\varepsilon / \varepsilon_{0}=10.5$.

band gaps (with respect to the geometric parameters $d / a$ and $r / a)$ are listed in Table VI. All the relative differences of the upper and lower edges are within $1.2 \%$. Figure 10 shows the corresponding plot of the band structure at a smaller $\varepsilon / \varepsilon_{0}$ of 10.5. The optimal full band gap is apparently simultaneous, and, as for $\varepsilon / \varepsilon_{0}=11.4$, has band edges occurring at $M-\Gamma$ (upper-lower) for $H$ polarization and at $\Gamma-X$ (upper-lower) for $E$ polarization. Likewise, for the hexagonal lattice of type TRI1, we vary the dielectric contrast $\varepsilon / \varepsilon_{0}$ from 12 to 14 , centered at 13 , and the results for the optimal full band gaps (with respect to the geometric parameters $d / a$ and $r / a$ ) are listed in Table VII. Although the relative differences between the upper edges are basically within $0.18 \%$, the relative difference between the lower edges tend to increase with decreasing dielectric contrast: $\beta_{\text {low }}=4.5 \%$ and $4.6 \%$ for $\varepsilon / \varepsilon_{0}=12.5$ and 12 , respectively. Figure 11 shows a plot of the band structure at a large $\varepsilon / \varepsilon_{0}$ of 14 . The optimal full band gap is apparently simultaneous, and, as for $\varepsilon / \varepsilon_{0}=13$, $H$ polarization has band edges at $M-K$ (upper-lower), while $E$ polarization has band edges at $K-\Gamma$ (upper-lower).

From the above results, we have the following observations. In spite of different possibilities for balancing the band edges, two geometric parameters of photonic crystals are quite effective for optimizing band gap by giving simultaneous band edges of $E$ and $H$ polarizations. It is important to

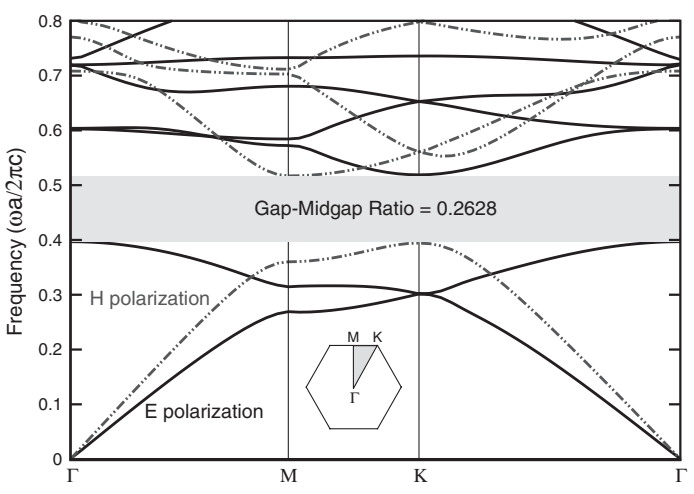

Fig. 11. Band structures computed with $256 \times 256$ grid for type TRI1 in Fig. 5(d). The optimal band gap is attained at $r / a=0.23$ and $d / a=0.02$ with $\varepsilon / \varepsilon_{0}=14$.

note that this is by no means a proof or claims of proof of a rigorously valid theorem, but it does provide an interesting observation and may serve as an empirical criterion in finding two-dimensional photonic structures with optimal (and largest) full band gaps. On the other hand, we observed cases with some larger relative differences: those in Tables $\mathrm{V}$ and VII with a $\beta_{\text {low }}$ larger than $2 \%$ but within $5 \%$. This indicates that there are certain possibilities for two geometric parameters to be not sufficient or for other combinations of parameters to be more adequate. It is indicated here that the dielectric contrast $\varepsilon / \varepsilon_{0}$ plays an important role in band gaps. Two geometric parameters would not allow simultaneous band gaps if the dielectric contrast $\varepsilon / \varepsilon_{0}$ decreases close to 1 .

It is also of interest to take a look at the values of the two geometric parameters when the optimal full band gaps occur. Table VIII lists the optimal gap-midgap ratios and corresponding geometric parameters for the three types of square lattice in Fig. 4 with $\varepsilon / \varepsilon_{0}=11.4$. Although they belong to the same group of symmetry, the two geometric parameters vary significantly in magnitude. For example, the radius $r / a$ varies from 0.275 for type SS2 to 0.31 for type SCR, and the filling fraction $f$ varies from $31.5 \%$ for type SS2 to $37.8 \%$ for type SS1. Type SCR has the largest full band gap in terms of the gap-midgap ratio. Table IX lists the optimal 
Table VIII. Optimal gap-midgap ratios and corresponding geometric parameters for the square lattice in Fig. 4 with $\varepsilon / \varepsilon_{0}=11.4$. $f$ is the filling fraction of the dielectric material.

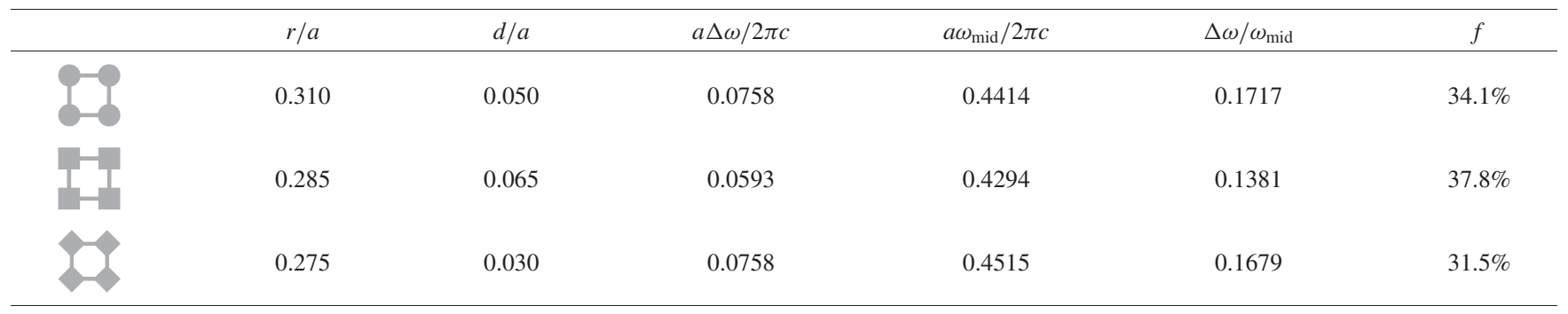

Table IX. Optimal gap-midgap ratios and corresponding geometric parameters for the hexagonal lattice in Fig. 5 with $\varepsilon / \varepsilon_{0}=13$. $f$ is the filling fraction of the dielectric material.

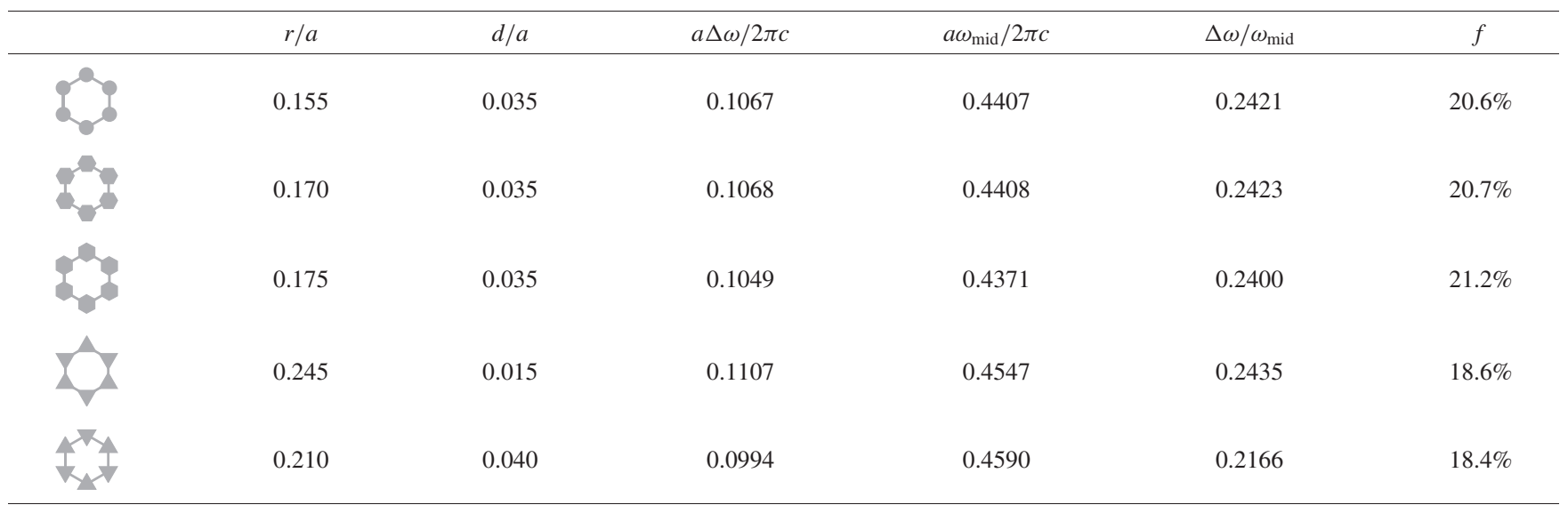

Table X. Large full band gaps associated with various geometries in the literature.

\begin{tabular}{|c|c|c|c|c|c|}
\hline & shape & $\varepsilon / \varepsilon_{0}$ & $a \omega_{\text {mid }} / 2 \pi c$ & $a \Delta \omega / 2 \pi c$ & $\Delta \omega / \omega_{\mathrm{mid}}$ \\
\hline 11) & & 13 & & & 0.186 \\
\hline 15) & & 11.4 & $(0.71)$ & 0.1158 & $(0.1631)$ \\
\hline 16) & & 12.25 & & 0.0872 & 0.182 \\
\hline 17) & & 11.4 & $(\sim 0.45)$ & 0.0762 & $(\sim 0.1693)$ \\
\hline 18) & & 12.96 & & 0.090 & 0.171 \\
\hline 19) & 88 & tellurium & & 0.046 & 0.179 \\
\hline 20) & $\because 0$ & 11.4 & $>0.9$ & 0.0967 & $<0.1074$ \\
\hline & & & $>0.8$ & 0.0849 & $<0.1061$ \\
\hline
\end{tabular}

gap-midgap ratios and corresponding geometric parameters for the five types of hexagonal lattice in Fig. 5 with $\varepsilon / \varepsilon_{0}=13$. Again, in spite of the same group of symmetry, the geometric parameters vary significantly in magnitude. The radius $r / a$ varies from 0.155 for type HCR to 0.245 for type TRI1. The width $d / a$ has a small difference between 0.035 to 0.04 except for type TRI1, which has a value of 0.015. In particular, for type TRI1, when the radius $r$ of the circumcircle of the triangle (cf. Fig. 7) is larger than $a / 2 \sqrt{3}$, the dielectric columns already touch each other. As a result, the optimal $d / a$ for this case is relatively smaller than those for the other cases. The midgap frequency $\omega_{\text {mid }}$, however, has a small difference, ranging from 0.4371 for type HEX2 to 0.4590 for type TRI2. The filling fraction $f$ of the dielectric material ranges from $18.4 \%$ for type TRI2 to $21.2 \%$ for type HEX2. The four types HCR, HEX1, HEX2 and TRI1 all have significantly large gap-midgap ratios $\Delta \omega / \omega_{\text {mid }}$ near 0.24 , while type TRI 2 has a substantially smaller gap-midgap ratio of 0.2166 . For type TRI2, it is also noted that the coincidence of the band edges is the worst (cf. Table V), namely, the full band gap is not simultaneous.

Finally, a comparison of the full band gap size with those of previous studies that reported large full band gaps is available. Table $\mathrm{X}$ lists the references that reported the 


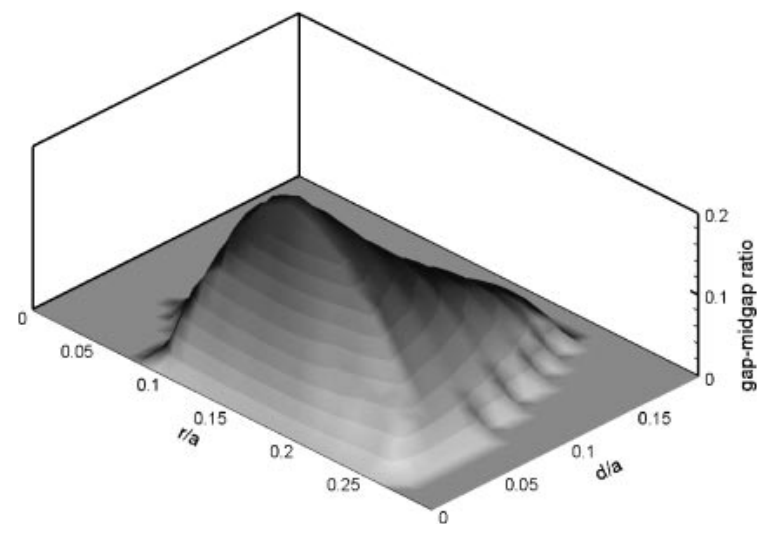

Fig. 12. A three-dimensional terrain of the major full band gaps for type HEX1 in Fig. 5(b) with $\varepsilon / \varepsilon_{0}=13$.

numerics of large band gaps for various photonic structures. At $\varepsilon / \varepsilon_{0}=11.4$ (GaAs-air), we observe from Tables VIII and $\mathrm{X}$ that type SCR has the largest full band gap. In particular, we notice from Tables IX and X that the largest optimal gap-midgap ratio obtained in the present study for the hexagonal lattice with $\varepsilon / \varepsilon_{0}=13$ (silicon-air) is $30 \%$ larger than the largest reported elsewhere..$^{11,15-20)}$ In this study, to ensure the global optimum of the full band gap with respect to the geometric parameters, a terrain plot is drawn for visualization in each case. Figure 12 shows an example for type HEX1 with $\varepsilon / \varepsilon_{0}=13$ : there are a total of 60024 eigenfrequencies computed for this diagram.

\section{Concluding Remarks}

This study is an attempt to address the criterion when photonic structures have the optimized largest band gaps. We start by showing how a photonic structure with one single parameter can be easily modified to give a substantial larger full band gap with simultaneous band edges. The basic thought is that (at least) two geometric parameters are needed for appropriately balancing the band edges of $E$ and $H$ polarizations to give simultaneous maximum band gaps. For this purpose, we have investigated a class of photonic structures with a square lattice and another class with a hexagonal lattice; both have two geometric parameters associated with dielectric columns and connecting rods. Each class is constructed to have the same group of symmetry, and the photonic structures in the same class have band edges for $E$ and $H$ polarizations occurring at the same Bloch wave numbers. The two classes of photonic crystals have the following feature: the optimal (and the largest) full band gap is obtained when both band gaps for $E$ and $H$ polarizations have simultaneous band edges. In addition, significantly larger gap-midgap ratios were obtained for the proposed classes of photonic structures, than those in the literature. Nevertheless, the feature is limited to two-dimensional photonic crystals and has no obvious extendsion to three-dimensional crystals. On the other hand, the extension of the present study to metallodielectric photonic crystals is currently under extensive investigation.

\section{Acknowledgements}

This work was supported in part by the National Science Council of the Republic of China under Contract No. NSC 91-2212-E-002-072, and the Ministry of Economic Affairs of the Republic of China under Contract No. MOEA 92-EC17-A-08-S1-0006.

1) E. Yablonovitch: Phys. Rev. Lett. 58 (1987) 2059.

2) S. John: Phys. Rev. Lett. 58 (1987) 2486.

3) N. Garcia and A. Z. Genack: Phys. Rev. Lett. 66 (1991) 1850.

4) S. John: Phys. Today May (1991) 32.

5) S. Lin and G. Arjavalingam: J. Opt. Soc. Am. B 11 (1994) 2124.

6) T. F. Krauss, R. M. De La Rue and S. Brand: Nature 383 (1996) 699.

7) J. S. Foresi, P. R. Villeneuve, J. Ferrera, E. R. Thoen, G. Steinmeyer, S. Fan, J. D. Joannopoulos, L. C. Kimerling, H. I. Smith and E. P. Ippen: Nature 390 (1997) 143.

8) A. Mekis, J. C. Chen, I. Kurland, S. Fan, P. R. Villeneuve and J. D. Joannopoulos: Phys. Rev. Lett. 77 (1996) 3787.

9) O. Painter, R. K. Lee, A. Scherer, A. Yariv, J. D. OBrien, P. D. Dapkus and I. Kim: Science 284 (1999) 1819.

10) D. L. Bullock, C. Shih and R. S. Margulies: J. Opt. Soc. Am. B 10 (1993) 399.

11) J. D. Joannoupoulos, R. D. Meade and J. N. Winn: Photonic Crystals (Princeton University Press, Princeton, 1995).

12) R. L. Chern, C. C. Chang, C. C. Chang and R. R. Hwang: Phys. Rev. E 68 (2003) 026704.

13) R. D. Meade, A. M. Rappe, K. D. Brommer, J. D. Joannopoulos and O. L. Alherhand: Phys. Rev. B 48 (1993) 8434.

14) S. G. Johnson and J. D. Joannopoulos: Photonic Crystals: The Road from Theory to Practice (Kluwer Academic, Boston, 2002).

15) L. Shen, S. He and S. Xiao: Phys. Rev. B 66 (2002) 165315.

16) N. Susa: J. Appl. Phys. 91 (2002) 3501.

17) M. Qiu and S. He: J. Opt. Soc. Am. B 17 (2000) 1027.

18) X. H. Wang, B. Y. Gu, Z. Y. Li and G. Z. Yang: Phys. Rev. B 60 (1999) 11417.

19) Z. Y. Li, B. Y. Gu and G. Z. Yang: Phys. Rev. Lett. 81 (1998) 2574.

20) C. M. Anderson and K. P. Giapis: Phys. Rev. Lett. 77 (1996) 2949. 